\title{
Nivel del magnesio sérico y su relación con el medio interno en pacientes incidentes al servicio de emergencia médica de un hospital general
}

\author{
Serum magnesium level and its relationship with internal medium in incident patients attending an \\ emerging service of a public hospital
}

Luis Enrique Barrios Juárez ${ }^{1, \mathrm{~b}}$, Renzo Javier Ortega Bazalar ${ }^{1, \mathrm{~b}}$, Juan Carlos Vega Quiñones ${ }^{1, \mathrm{~b}}$, Javier Antonio Cieza Zevallos ${ }^{1, a ; 2, c}$

\section{RESUMEN}

El magnesio participa en múltiples procesos metabólicos y diversos estudios han mostrado que sus niveles alterados se asocian a enfermedades crónicas, alteraciones del medio interno y en unidades de cuidados críticos, está asociado a mayor mortalidad. Objetivos: Determinar la relación entre el nivel de magnesio sérico y las alteraciones del medio interno, en pacientes incidentes al servicio de emergencia de un hospital general de Lima, Perú. Material y métodos: Se incluyeron 201 pacientes con medida del magnesio, creatinina y electrolitos séricos, así como gasometría arterial al ingreso a emergencia del Hospital Cayetano Heredia (HCH) durante los años 2017-2018. Se analizó los valores del magnesio sérico y su asociación con las demás variables electrolíticas y clínicas. Resultados: La edad media de la población fue $60,1 \pm 20,1$ años. El magnesio sérico promedio fue 2,05 $\pm 0,57 \mathrm{mg} / \mathrm{dl}$. La hipomagnesemia e hipermagnesemia tuvieron una frecuencia de $20,4 \%$ y $21,9 \%$ respectivamente. La regresión lineal bivariado mostró

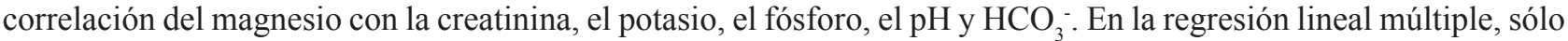
el pH se asoció significativamente con el magnesio. En el análisis del magnesio estratificado, el pH mostró la mayor significancia estadística. No hubo relación con la edad, sexo y variables clínicas. Conclusión: El magnesio es una alteración frecuente en la población que acude a la emergencia del $\mathrm{HCH}$ y tiene correlación con el bicarbonato, la creatinina, el potasio, el fósforo y el $\mathrm{pH}$; siendo el último la variable más relevante.

PALABRAS CLAVE: Magnesio, electrólitos, equilibrio ácido-base. (Fuente: DeCS BIREME).

\section{SUMMARY}

Serum magnesium participates in multiple metabolic processes; several studies have shown that alterations in its serum levels are associated with chronic illnesses, alterations in the internal medium and the critical care units these alterations are associated with higher mortality. Objectives: To determine the relationship between serum magnesium level and alterations in the internal medium in incident patients attending an emerging service of a public hospital. Methods: 201 patients were included who had measurements of serum magnesium, creatinine, serum electrolytes and blood gases on admission to the emergency room at Hospital Cayetano Heredia (CHH)

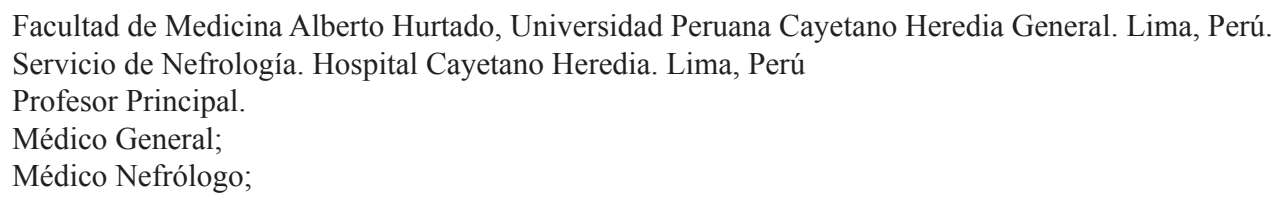


between 2017-2018. Results: Mean age was $60.1 \pm 20.1$ years. Mean serum magnesium level was $2.05 \pm 0.57$ $\mathrm{mg} / \mathrm{dl}$. Hypo and hypermagnesemia were observed in $20.4 \%$ and $21.9 \%$, respectively. Bivariate lineal analysis showed correlation of magnesium with creatinine, potassium, phosphorus, $\mathrm{pH}$ and bicarbonate. The multiple lineal regression showed association of magnesium with $\mathrm{pH}$ only, which was corroborated in the stratified analysis, no association with age, gender and clinical variables was found. Conclusions: Serum magnesium levels are frequently altered in patients attending the emergency room at $\mathrm{CHH}$ and correlates with bicarbonate, creatinine, potassium, phosphorus and $\mathrm{pH}$, with the latter being more relevant.

KEYWORDS: Magnesium, electrolytes, acid-base equilibrium. (Source: MeSH NLM).

\section{INTRODUCCIÓN}

El magnesio $(\mathrm{Mg})$ es un catión divalente almacenado principalmente en los huesos, siendo mínima la cantidad a nivel extracelular. Cumple un rol importante en procesos fisiológicos encargados del almacenamiento, transferencia y uso de energía; tiene relevancia en el metabolismo del calcio y potasio, participa en la síntesis de proteínas y además, permite el flujo de electrolitos a través de las membranas de los tejidos cardíaco y nervioso, promoviendo el equilibrio ácido base a nivel del medio interno (1-5).

El nivel sérico normal del magnesio oscila entre 1,7 y $2,2 \mathrm{mg} / \mathrm{dl}$; se define hipomagnesemia e hipermagnesemia como la concentración sérica de magnesio menor o mayor, respectivamente (4).

Se ha descrito que niveles bajos de magnesio sérico han sido asociados a enfermedades crónicas como diabetes mellitus tipo 2, enfermedad de Alzheimer, hipertensión arterial, accidentes cerebrovasculares, enfermedad cardiovascular y síndrome metabólico $(4,6,7)$. Además, investigaciones muestran que existe asociación entre la concentración sérica del magnesio y ciertas variables del medio interno. Moskowitz y col. (8), demostraron que pacientes con hipomagnesemia presentaban mayor riesgo de desarrollar acidosis láctica, esta asociación era de mayor magnitud en pacientes diabéticos y alcohólicos.

Múltiples estudios han evaluado los niveles séricos de magnesio en pacientes en unidades de cuidados críticos, concluyendo que tanto la hipomagnesemia como la hipermagnesemia están asociados a mayor mortalidad (9-11).

Estudios en poblaciones urbanas de Holanda e Irán, describen una prevalencia entre 2 a $4 \%$ de niveles de magnesio alterados $(12,13)$. Sin embargo, Mejía F. (14), en den México, en 5410 participantes, reportó una prevalencia de hipomagnesemia de $36,1 \%$ en población general no hospitalizada (14). Whang y Reider (15), determinaron que solo el $10 \%$ de pacientes con hipomagnesemia eran diagnosticados debidamente, siendo el $90 \%$ sub diagnosticados.

Es oportuno estudiar este ion en una población que mayormente acude en situación de desnutrición al Hospital Cayetano Heredia $(\mathrm{HCH})$ de la ciudad de Lima, situación observada por Cieza y col. (16), quienes reportaron que $81,5 \%$ de pacientes adultos incidentes al servicio de emergencia presentaban algún grado de hipoalbuminemia.

El objetivo de este estudio fue determinar si existe relación entre el nivel de magnesio sérico y las alteraciones del medio interno, en pacientes incidentes al servicio de emergencia de un hospital general de Lima, Perú.

\section{MATERIAL Y MÉTODOS}

Estudio descriptivo y transversal realizado en pacientes incidentes al servicio de emergencia del HCH, entre enero de 2017 a febrero del 2018.

El tamaño de muestra fue calculado en 207 pacientes, se realizó en función de una observación preliminar que mostró que $65,5 \%$ de pacientes en el Servicio de Emergencia médica del $\mathrm{HCH}$, tenían valores considerados normales de magnesio sérico, aceptando un error típico de 5\% y una confianza del $95 \%$ en una población potencialmente infinita.

Los criterios de inclusión fueron: Pacientes incidentes recientes a la emergencia del $\mathrm{HCH}$, edad mayor o igual a 18 años y contar obligatoriamente con medición de magnesio sérico, creatinina sérica, electrolitos séricos y estudios de gasometría sanguínea. Las variables registradas fueron: edad, sexo; creatinina, magnesio y electrolitos séricos, gases arteriales, fósforo y calcio sérico total. Otras variables clínicas consideradas fueron la presión arterial, frecuencia 
cardíaca y respiratoria, reflejos osteotendinosos, Glasgow, alteraciones neuromusculares y existencia de sepsis.

Las variables del medio interno fueron tratadas en forma cuantitativa como valores numéricos y las variables clínicas como categóricas. Los disturbios de la gasometría arterial fueron clasificados como acidosis metabólica, acidosis respiratoria, alcalosis metabólica y alcalosis respiratoria. El magnesio sérico fue tratado cuantitativamente como valor numérico continuo y cualitativamente como variable estratificada según criterio de los valores normales, inferiores y superiores aceptados en la literatura.

Inicialmente se describe la población en función de los valores de magnesio y posteriormente un análisis bivariado consideró el magnesio como variable independiente y las diferentes variables del medio interno como variables de asociación. Posteriormente, se estudió la asociación significativa de las variables mediante un modelo de ANOVA.

El trabajo de investigación fue aprobado por el Comité Institucional de Ética de Investigación (CIEI) de la Universidad Peruana Cayetano Heredia y el CIEI del $\mathrm{HCH}$

\section{RESULTADOS}

Del total de 207 historias clínicas revisadas, 201 fueron incluidas por contar con los criterios de inclusión exigidos y verificados, seis no fueron incluidos por tener edad menor a los 18 años.

La edad media de la población estudiada fue 60,1 \pm 20,1 años; 104 (51,7\%) varones y 98 (48,3\%), mujeres. El IMC promedio fue $24,53 \pm 3,91 \mathrm{~kg} / \mathrm{m}^{2}$ y la SC 1,65 $\pm 0,23 \mathrm{~m}^{2}$. El promedio del magnesio sérico fue 2,05 $\pm 0,57 \mathrm{mg} / \mathrm{dl}$, pero el $42,3 \%$ de la población estudiada presentó valores alterados, siendo la frecuencia de la hipomagnesemia 20,4\% y de hipermagnesemia 21,9\%. El disturbio ácido base más frecuente hallado fue la alcalosis respiratoria $(63,1 \%)$.

El análisis de regresión lineal simple mostró que los valores de magnesio correlacionaron con la creatinina, el potasio, el fósforo, el $\mathrm{pH}$ y el bicarbonato $\left(\mathrm{HCO}_{3}^{-}\right)$ sérico (tabla 1). Al realizar el análisis de la regresión lineal múltiple, el modelo final mostró que el $\mathrm{pH}$ fue la única variable relacionada significativamente con el magnesio (tabla 2).

Al considerar el magnesio como variable cualitativa, el ANOVA mostró que el pH, el bicarbonato, el cloro, la creatinina, el potasio y el fósforo alcanzaron significancia estadística (tabla 3). El análisis post hoc mediante Bonferroni demostró que en relación al potasio y el bicarbonato, la diferencia fue significativa solamente entre los grupos con hipomagnesemia e hipermagnesemia, pero respecto al cloro la diferencia significativa estuvo entre los grupos con normomagnesemia e hipomagnesemia. Al evaluar el magnesio estratificado con el pH, se evidenció

Tabla 1. Correlación lineal bivariada del magnesio sérico con las diversas variables del medio interno.

\begin{tabular}{|c|c|c|c|c|c|c|c|c|c|}
\hline & $\begin{array}{c}\text { Creatinina } \\
(\mathrm{mg} / \mathrm{dl})\end{array}$ & $\begin{array}{l}\text { Potasio } \\
(\mathrm{mEq} / \mathrm{l})\end{array}$ & $\begin{array}{c}\text { Sodio } \\
(\mathrm{mEq} / \mathrm{l})\end{array}$ & $\begin{array}{c}\text { Cloro } \\
(\mathrm{mEq} / \mathrm{l})\end{array}$ & $\begin{array}{c}\text { Calcio } \\
\text { (mg/dl) }\end{array}$ & $\begin{array}{l}\text { Fósforo } \\
\text { (mg/dl) }\end{array}$ & ClCr* & pH & $\begin{array}{l}\text { HCO3- } \\
(\mathrm{mEq} / \mathrm{l})\end{array}$ \\
\hline$r$ & 0,185 & 0,172 & $-0,06$ & $-0,006$ & 0,009 & 0,192 & $-0,108$ & $-0,427$ & $-0,253$ \\
\hline $\mathrm{p}$ & 0,008 & 0,015 & 0,397 & 0,932 & 0,919 & 0,019 & 0,143 & 0,000 & 0,000 \\
\hline $\mathrm{n}$ & 201 & 201 & 201 & 201 & 120 & 149 & 186 & 201 & 201 \\
\hline
\end{tabular}

* $\mathrm{ClCr}=$ Aclaramiento de creatinina, $\mathrm{ml} / \mathrm{min}$.

Tabla 2. Regresión lineal múltiple de las variables del medio interno relacionadas significativamente con el magnesio sérico en la regresión bivariada.

\begin{tabular}{lccc}
\hline \multicolumn{1}{c}{ Modelo } & $\begin{array}{c}\text { Coeficientes }(\boldsymbol{\beta}) \mathbf{~ n o} \\
\text { estandarizados } \pm \mathbf{E E}\end{array}$ & $\begin{array}{c}\text { Coeficientes }(\boldsymbol{\beta}) \\
\text { tipificados }\end{array}$ & $\boldsymbol{p}$ \\
\hline Constante) & $25,17 \pm 4,71$ & & 0,000 \\
Potasio $(\mathrm{mEq} / \mathrm{l})$ & $0,001 \pm 0,058$ & 0,002 & 0,984 \\
Creatinina $(\mathrm{mg} / \mathrm{dl})$ & $0,005 \pm 0,011$ & 0,045 & 0,639 \\
Fósforo $(\mathrm{mg}(\mathrm{dl})$ & $0,006+0,010$ & 0,017 & 0,846 \\
pH & $-3,151 \pm 0,029$ & $-0,485$ & 0,000 \\
Bicarbonato $(\mathrm{mEq} / \mathrm{l})$ & $0,008 \pm 0,010$ & 0,083 & 0,400 \\
\hline
\end{tabular}


Tabla 3. Análisis de la varianza del magnesio con otras variables del medio interno.

\begin{tabular}{|c|c|c|c|c|c|}
\hline & & $\begin{array}{c}\text { ANOVA } \\
\text { gl }\end{array}$ & $\begin{array}{l}\text { Media } \\
\text { cuadrática }\end{array}$ & $\mathbf{F}$ & $\mathbf{p}$ \\
\hline \multirow[t]{3}{*}{ Creatinina } & Inter-grupos & 2 & 186,7 & 8,46 & 0,000 \\
\hline & Intra-grupos & 198 & 22,1 & & \\
\hline & Total & 200 & & & \\
\hline \multirow[t]{3}{*}{ Sodio } & Inter-grupos & 2 & 146,2 & 1,52 & 0,221 \\
\hline & Intra-grupos & 198 & 96,2 & & \\
\hline & Total & 200 & & & \\
\hline \multirow[t]{3}{*}{ Potasio } & Inter-grupos & 2 & 6,1 & 7,59 & 0,001 \\
\hline & Intra-grupos & 198 & 0,80 & & \\
\hline & Total & 200 & & & \\
\hline \multirow[t]{3}{*}{ Cloro } & Inter-grupos & 2 & 227,6 & 3,98 & 0,020 \\
\hline & Intra-grupos & 198 & 57,1 & & \\
\hline & Total & 200 & & & \\
\hline \multirow[t]{3}{*}{ Calcio } & Inter-grupos & 2 & 0,01 & 0,002 & 0,998 \\
\hline & Intra-grupos & 117 & 5,9 & & \\
\hline & Total & 119 & & & \\
\hline \multirow[t]{3}{*}{ Fósforo } & Inter-grupos & 2 & 20,5 & 6,15 & 0,003 \\
\hline & Intra-grupos & 146 & 3,3 & & \\
\hline & Total & 148 & & & \\
\hline \multirow{3}{*}{$\begin{array}{l}\text { Aclaramiento } \\
\text { Creatinina }\end{array}$} & Inter-grupos & 2 & 10344,5 & 2,47 & 0,087 \\
\hline & Intra-grupos & 183 & 4183,6 & & \\
\hline & Total & 185 & & & \\
\hline \multirow[t]{3}{*}{ pH } & Inter-grupos & 2 & 0,09 & 12,31 & 0,000 \\
\hline & Intra-grupos & 198 & 0,01 & & \\
\hline & Total & 200 & & & \\
\hline \multirow[t]{3}{*}{ Bicarbonato } & Inter-grupos & 2 & 199,2 & 5,87 & 0,003 \\
\hline & Intra-grupos & 198 & 34,0 & & \\
\hline & Total & 200 & & & \\
\hline \multirow[t]{3}{*}{ PCO2 } & Inter-grupos & 2 & 67,0 & 1,12 & 0,328 \\
\hline & Intra-grupos & 198 & 59,8 & & \\
\hline & Total & 200 & & & \\
\hline
\end{tabular}

diferencia estadísticamente significativa en todos los grupos al igual que con el fósforo, pero fue mucho más relevante para el $\mathrm{pH}$.

La regresión logística multivariada mostró que el $\mathrm{pH}$ fue la única variable con significancia estadística. El mejor modelo con el que el pH se relacionó con el magnesio fue una correlación cúbica (gráfico 1).

Respecto al equilibrio ácido-base, la acidosis metabólica fue el disturbio predominante en los pacientes con hipermagnesemia $(47,2 \%)$, mientras que la alcalosis respiratoria fue la predominante en los pacientes con hipomagnesemia $(56,6 \%)$ y en los pacientes con normomagnesemia $(57,4 \%)$. No se observó alcalosis metabólica en pacientes con hipermagnesemia. La acidosis metabólica y respiratoria son los disturbios que van en aumento con el nivel del magnesio sérico, siendo la acidosis metabólica la que presentó mayor incremento (gráfico 2). La alcalosis metabólica fue la única variable que no mostró significancia estadística respecto a las categorías del magnesio sérico. 


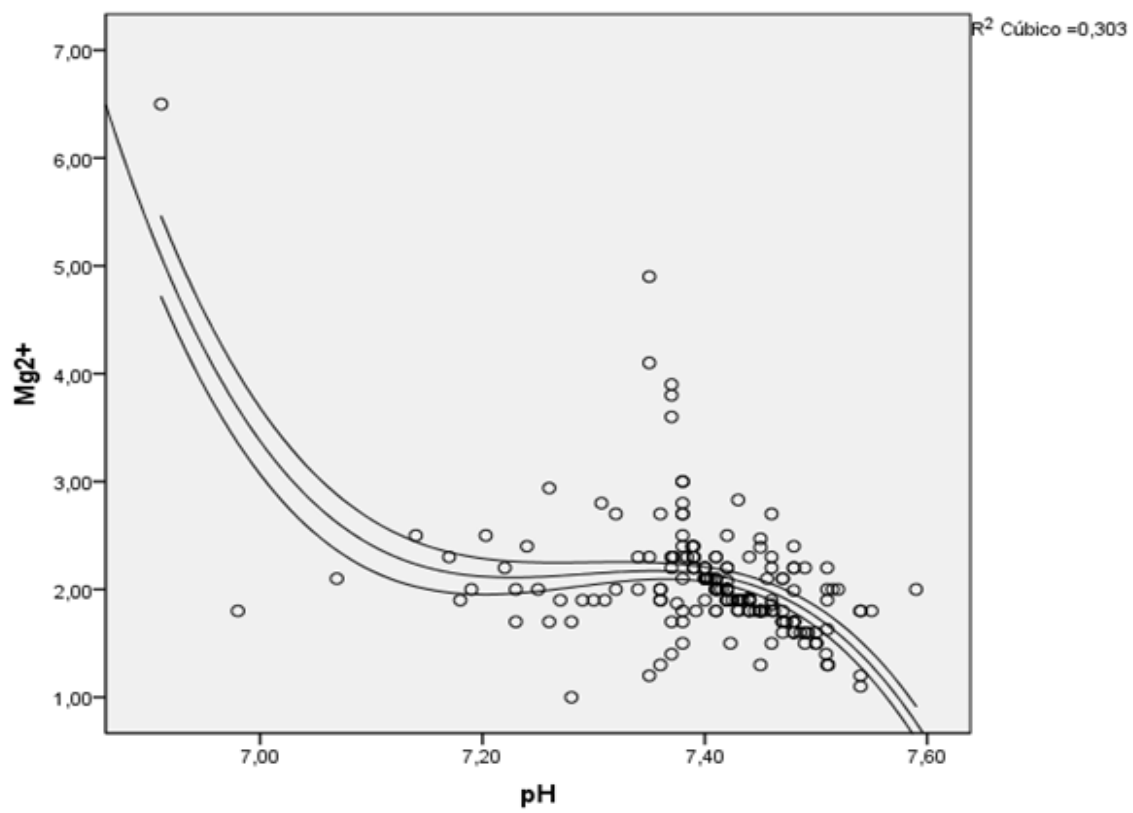

Gráfico 1. Modelo de magnesio en función del pH.

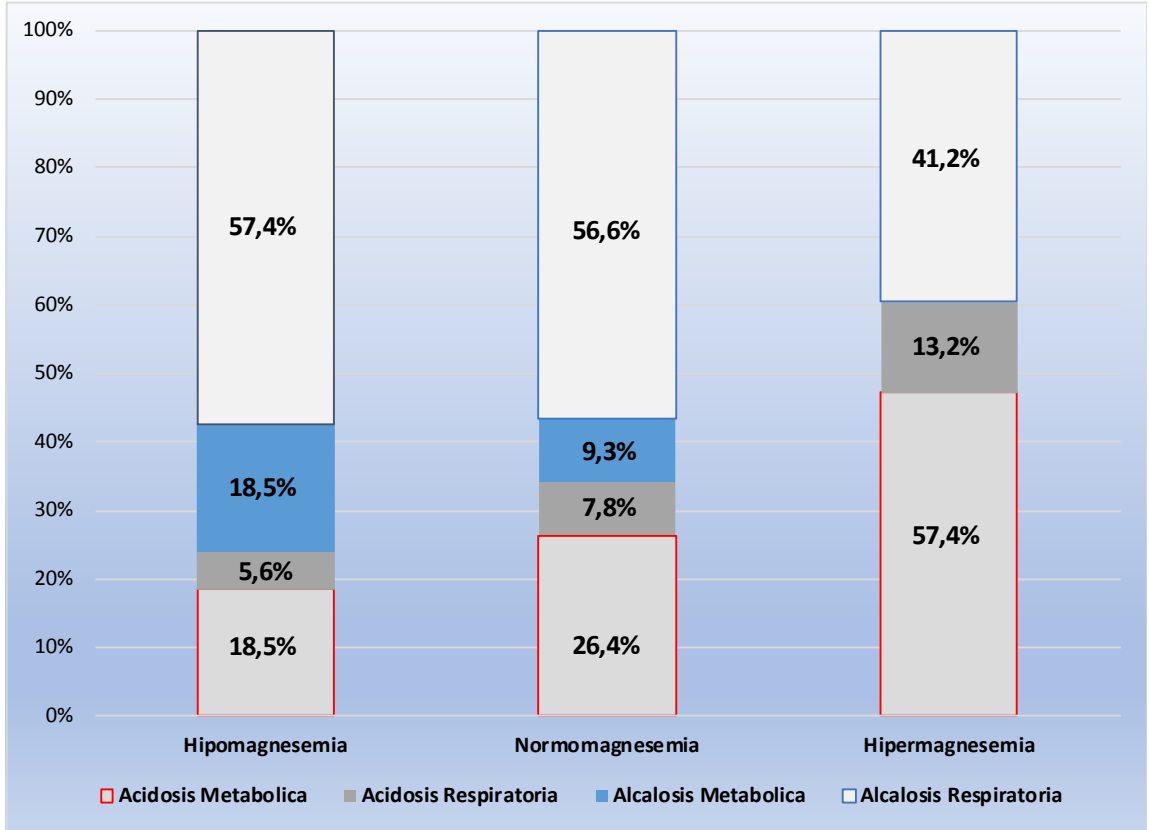

Gráfico 2. Presencia de disturbios de la gasometría arterial en la población estudiada.

No se encontró asociación estadísticamente significativa con las variables clínicas consideradas en este estudio, ni con la edad y ni con el sexo.

\section{DISCUSIÓN}

En el presente estudio se encontró que el $42,3 \%$ de los pacientes presentaron alguna alteración del magnesio sérico al ingreso al servicio de emergencia sin haber recibido algún tratamiento médico hospitalario (hipomagnesemia 20,4\% e hipermagnesemia 21,9\%). Esto implica que, la población general en nuestro medio presenta alguna alteración previa del magnesio sérico (posiblemente asintomática). Este hallazgo se corrobora con el estudio de Mejía et al. (14), realizado en México en el que se reporta hipomagnesemia en un rango de 31,2 a $40,2 \%$ en mujeres y 23,9 a $39,7 \%$ en varones. Sin embargo, contrasta con lo observado 
en población holandesa en donde alrededor del $6 \%$ presenta hipomagnesemia (12). Subrayamos que ambos estudios fueron realizados en población no hospitalaria.

No se observó asociación entre los niveles de magnesio sérico y el sexo, como también concluyen Bertinato et al. (17), en un estudio realizado en una población canadiense; sin embargo, encontraron que la edad era el predictor más fuerte de la concentración de magnesio, mientras que en nuestro estudio no se encontró este hallazgo (17).

Nuestro estudio determinó la asociación entre el magnesio y el bicarbonato, la creatinina, el fósforo, el potasio y el $\mathrm{pH}$ sérico. Esto se puede observar principalmente en pacientes con enfermedad renal crónica (ERC) en estadios avanzados en los cuales los valores de creatinina, fósforo y potasio tienden al aumento por disminución de la tasa de filtración glomerular (18). Sin embargo, la variable aclaramiento de creatinina $(\mathrm{ClCr})$ no tuvo significancia estadística, lo cual contrasta con lo observado en el estudio de Cunnighan, en el que se observa una relación inversamente proporcional entre el magnesio y el $\mathrm{ClCr}$. Esto podría deberse a que en nuestro estudio se realizó la estimación del $\mathrm{ClCr}$ mediante la fórmula de Crockfort-Gault, en la cual intervienen variables como el peso, edad y el sexo, que en poblaciones desnutridas como la aquí estudiada puede generar sesgos (19).

Además, se determinó que el $\mathrm{pH}$ fue la variable más relacionada al magnesio: a mayor concentración de hidrogeniones, mayor concentración del magnesio sérico. Esto es explicable porque en este estudio se incluyeron pacientes con ERC, en los cuales existe disminución de la excreción de amonio y reabsorción de bicarbonato. Por ende, se produce un aumento de los hidrogeniones (20), y la ERC está relacionada con aumento del valor de magnesio sérico (18).

El valor promedio de la creatinina sérica varió según la concentración de magnesio, los valores de creatinina más altos se relacionaron con hipermagnesemia. Llama la atención que la hipomagnesemia tuvo un nivel de creatinina ligeramente elevado con una media de $2,1 \mathrm{mg} / \mathrm{dl}$, y niveles séricos de magnesio normales tienen también niveles séricos de creatinina elevados con una media de 3,1. Sobre este punto, un estudio retrospectivo de Cheungpasitporn $\mathrm{W}$ et al. (21), mostró que existe asociación de los niveles séricos de magnesio y riesgo de lesión renal aguda al momento de hospitalización por existir injuria renal aguda. Además, el mismo autor comenta que existe una mayor incidencia de injuria renal aguda en asociación con hipomagnesemia e hipermagnesemia. Sin embargo, nuestro estudio incluyó pacientes con ERC, lo cual justificaría un aumento del magnesio sérico. Usualmente hasta que no hay reducción severa en la GFR ( $<30 \mathrm{ml} / \mathrm{min})$, los niveles séricos de $\mathrm{Mg}$ son normales; si hay niveles más bajos de función renal, el Mg sérico aumenta (22). Muchos de nuestros pacientes son desnutridos y valores no muy elevados de creatinina y a veces hasta en niveles considerados normales puede existir disfunción renal que explicaría el aumento del magnesio sérico tempranamente.

En conclusión, es frecuente encontrar alteraciones en los niveles de magnesio sérico en la población que acude al servicio de emergencia médica del Hospital Cayetano Heredia antes de cualquier intervención médica. Se evidencia correlación entre el magnesio y la creatinina, el potasio, el fósforo, el bicarbonato y el $\mathrm{pH}$; siendo este último el que presenta mayor relevancia, situación que debe ser considerada por el médico de nuestras emergencias donde existe una alta frecuencia de pacientes desnutridos, situación que conlleva el potencial riesgo de subestimar las alteraciones de este importante ion.

Se recomienda que en los servicios de emergencia de los hospitales nacionales que atienden pacientes de cierta complejidad, medir el magnesio sérico, debería ser considerado tan importante como los electrolitos (sodio y potasio) y la gasometría sanguínea.

\section{Declaración de financiamiento y de conflictos de interés:}

El estudio fue financiado por los autores. No existió conflictos de interés real, potencial o evidente, con los autores, incluyendo interés financiero o de otra índole.

\section{Contribución de autoría:}

LEBJ, RJOB y JCVQ: Participó en el diseño, recolección de datos, análisis e informe final del estudio; RO: Participó en el diseño, recolección de datos, análisis e informe final del estudio. JACZ: Participó en el diseño, análisis de los datos e informe final del estudio.

\section{Correspondencia:}

\section{Javier Cieza}

Correo electrónico: javier.cieza@upch.pe 


\section{REFERENCIAS BIBLIOGRÁFICAS}

1. Hall JE. Organismo funcional del cuerpo humano y control del medio interno. EN: Bartolomé Bonet. Tratado de Fisiología Médica Vol. 1. 11a ed. España: Elseiver; 2011. p. 3-9.

2. Baaij J, Hoenderop J, Bindels R. Magnesium in Man: Implications for Health and Disease. Physiol Rev. 2015; 95(1):1-46.

3. Jahnen-Dechent W, Ketteler M. Magnesium basics. Clinical Kidney Journal. 2012;5(1):3-14.

4. Volpe S. Magnesium in disease prevention and overall health. Adv Nutr. 2013; 4(3):378S-83S. doi: 10.3945/ an.112.003483.

5. Berend K, de Vries A, Gans R. Physiological Approach to Assessment of Acid-Base Disturbances. N Engl J Med. 2014; 371(15):1434-45.

6. Veronese N, Zurlo A, Solmi M, et al. Magnesium Status in Alzheimer's disease. Am J Alzheimers Dis Other Demen. 2016;31(3):208-13. doi: $10.1177 / 1533317515602674$

7. Shaikh S, Karira KA. Magnesium deficiency in heart failure patients with diabetes mellitus. J Pak Med Asso. 2011: 61(9):901-03.

8. Moskowitz A, Lee J, Donnino MW, Mark R, Celi LA, Danziger J. The Association Between Admission Magnesium Concentrations and Lactic Acidosis in Critical Illness. J Intensive Care Med. 2016; 31(3):187-92. doi: 10.1177/0885066614530659.

9. Fairley J, Glassford N, Zhang L, Bellomo R. Magnesium status and magnesium therapy in critically ill patients: A systematic review. J Crit Care. 2015; 30(6):1349-58.

10. Escuela M, Guerra M, Añón J, Martínez-Vizcaíno V, Zapatero M, García-Jalón A et al. Total and ionized serum magnesium in critically ill patients. Intensive Care Med. 2004; 31(1):151-156.

11. Haider D, Lindner G, Ahmad S, et al. Hypermagnesemia is a strong independent risk factor for mortality in critically ill patients: Results from a cross-sectional study. Eur J Intern Med. 2015; 26(7):504-507.

12. Liamis G, Rodenburg E, Hofman A, Zietse R, Stricker B, Hoorn E. Electrolyte disorders in community subjects: Prevalence and risk factors. Am J Med. 2013; 126(3): 256-63.
13. Syedmoradi L, Ghasemi A, Zahediasl S, Azizi F. Prevalence of hypo- and hypermagnesemia in an Iranian urban population. Ann Hum Biol. 2010; 38(2):150-55.

14. Mejía F, Shamah T, Villalpando S, García A, Méndez I. Deficiencias de hierro, zinc, cobre y magnesio en adultos mexicanos. Encuesta Nacional de Salud y Nutrición 2006. Salud Pública Mex. 2013; 55(3):275-284.

15. Whang R. Frequency of hypomagnesemia and hypermagnesemia. JAMA. 1990; 263(22):3063-64.

16. Cieza J, Casillas A, Da Fieno A, Urtecho S. Asociación del nivel de albúmina sérica y alteraciones de los electrolitos, gases sanguíneos y compuestos nitrogenados en pacientes adultos incidentes del servicio de emergencia de un hospital general. Rev Med Hered. 2016; 27(4); 223-29.

17. Bertinato J, Wang KC, Hayward S. Serum Magnesium Concentrations in the Canadian population and associations with diabetes, glycemic regulation, and insulin resistance. Nutrients. 2017; 9(3); 296-308.

18. Dhondup T, Qian Q. Electrolyte and acid-base disorders in chronic kidney disease and end-stage kidney failure. Blood Purif. 2017; 43:179-188.

19. Cunningham J, Rodríguez M, Messa P. Magnesium in chronic kidney disease Stages 3 and 4 and in dialysis patients. Clin Kidney J. 2012; 5(1):i39-i51.

20. Kraut J, Madias N: Metabolic acidosis of CKD: An update. Am J Kidney Dis. 2016; 67:307-317.

21. Cheungpasitporn W, Thongprayoon C, Erickson S. Admission hypomagnesemia and hypermagnesemia increase the risk of acute kidney injury. Renal Failure. 2015; 37(7):1175-1179.

22. Lee S, Ryu J, Kim S, Ryu D, Kang D, Choi K. The relationship between magnesium and endothelial function in end-stage renal disease patients on hemodialysis. Yonsei Med J. 2016; 57(6):1446.

Recibido: 26/07/2018

Aceptado: 24/05/2019 